\title{
Aggressive Behaviors among 15-16-Year-Old Romanian High School Students: Results from Two Consecutive Surveys Related to Alcohol and Other Drug Use at the European Level
}

\author{
Adina Bucur ${ }^{1}$, Sorin Ursoniu ${ }^{1}\left(\mathbb{D}\right.$, Constantin Caraion-Buzdea ${ }^{1}$, Virgil Ciobanu ${ }^{1}$, \\ Silvia Florescu ${ }^{2}$ and Cristian Vladescu ${ }^{1,2, *}$ \\ 1 Department of Public Health, Victor Babes University of Medicine and Pharmacy, \\ 300172 Timisoara, Romania; bucur.adina@umft.ro (A.B.); sursoniu@umft.ro (S.U.); \\ ccaraion@umft.ro (C.C.-B.); ciobanu.virgil@umft.ro (V.C.) \\ 2 Management and Professional Development, The National School of Public Health, \\ 021253 Bucharest, Romania; sflorescu@snspms.ro \\ * Correspondence: cristian.vladescu@gmail.com; Tel.: +40-722-627036
}

Received: 24 April 2020; Accepted: 19 May 2020; Published: 22 May 2020

\begin{abstract}
The aim of this paper is to examine aggressive behaviors among Romanian high school students between 15 and 16 years old, to compare data in two national representative samples and to identify factors associated with physical fighting. This study investigates the association of selected factors (social, school performance and substance use) with physical fighting. A total of 2289 Romanian students were included in the 2007 database and 2770 in the 2011 database. This study revealed that $35.87 \%$ of the teenagers have taken part in a physical fight during the previous 12 months, as compared with the European average of 31.5\%. Romania has the highest prevalence of violent behavior by participating in a group bruising of an individual in both surveys, 2007 and 2011. A logistic regression analysis performed for the 2011 study revealed the following factors associated with physical fighting: binge drinking during the previous 30 days, male gender, serious problems with friends, parent(s) who do not know where and with whom the adolescents spend their evenings, poor parental caring, low school grades, and high truancy. A decrease in almost all aggressive behaviors was noticed in 2011, compared to 2007. These findings may be useful to support and guide policy makers regarding improvement and implementation of strategies to further prevent aggressive behaviors in teenagers.
\end{abstract}

Keywords: aggressive behavior; adolescents; victimization; bullying

\section{Introduction}

Aggression in high school students is a problem in many countries [1-8] and adolescents are especially vulnerable to its consequences [9]. Bullying, victimization and fighting illustrate different types of involvement in violence during adolescence. Bullying involves negative physical or verbal action that has hostile intent, causes distress to the victim and includes a power differential between bullies and their victims [6]. According to Olweus, it is also bullying when a person is teased repeatedly in a way he/she does not like. Victimization by bullying occurs when a person is made the recipient of aggressive behavior [10]. Typically, it is someone less powerful than the perpetrator. Fighting is an aggressive behavior and, in most cases, the people involved are of a similar age and equal strength. Demographic, social, academic achievement and substance use (alcohol drinking, tobacco smoking, drug use) have shown association with violent behavior in adolescents [11,12]. 
According to a report released in 2016, the prevalence of fighting among adolescents aged 15 from Europe and North America varies between 22\% and $69 \%$ in boys and between $9 \%$ and $25 \%$ in girls [13].

Physical fighting was strongly associated with alcohol consumption and drug use [14-16].

The social developmental model states that the youth behavior is learned through a continuous process starting from childhood. The social agents that play an important role in their behavioral development are families, schools, peers, communities [17-19].

Adolescents who maintain a stronger, healthier relationship with their families and their education are less likely to participate in unacceptable behaviors, such as violence [20,21].

The relationship with parents, including poor parental monitoring and low parental support, has also been mentioned as risk factors for violent behavior among adolescents [22,23].

Physical fighting has also been associated with poor peer relationships [24,25]. One of the most important factors in sculpting and defining the adolescent behavior represents the time they spend with their peers and the relationships they establish with them. Numerous studies have shown that adolescents tend to engage in similar behaviors as their peers (smoking, drinking, fighting and/or engaging in sexual behavior) [26-28]. Many adolescents have at least one friend that uses substances, but when most of their peers engage in behaviors such as drinking, smoking, or even illegal activities, the risk of them doing the same increases. While engagement in peer group activity is normative for adolescents, it is when a person has high support from peers and low support from parents, that substance use is particularly elevated [28].

Beside the immediate effects, bullying, victimization and fighting have long-term negative consequences for the bullies, victims, fighters and those who observe the interaction $[29,30]$. Some studies have shown that children who are bullies tend to still be bullies as adults. Additionally, an interesting observation is that adult bullies with children of their own tend to raise them as bullies [30]. Because it relates to students, school violence has received substantial media, research, and political attention [31]. In Romania, no systematic studies on aggressive behavior among high school students have been published so far.

According to the Health Behaviour in School-aged Children (HBSC) study conducted in Romania between 2005 and 2006 [32], $6 \%$ of the girls and 24\% of the boys aged 15 have been involved in a physical fight at least three times in the last 12 months. Another survey performed by HBSC between 2009 and 2010 [33] found that $4 \%$ of the girls and 19\% of the boys aged 15 have been involved in a physical fight at least three times in the last 12 months.

However, there is increased concern about violent behaviors amongst adolescents in the school setting and on the community level; hospital, primary health care and ambulatory data shows increased numbers for adolescent victims of aggressive behaviors [34,35]. The 2007 [36] and 2011 [37] European School Survey Project on Alcohol and Other Drugs (ESPAD), which examined 35 and 36 European countries, respectively, including Romania, provided an opportunity to study aggressive behavior in a large national sample.

The first aim of this paper is to examine patterns of aggressive behavior of 15-16-year old high school students in Romania, and compare the collected data in two national representative samples from 2007 and 2011. The second aim of the study is to identify factors (gender, social, behavioral and school performance) associated with physical fights in adolescents in Romania.

We hypothesized that physical fighting is associated with different types of factors, such as demographic (gender), social (relationship with parents and friends, parental control), school performance (grades), problem behavior (truancy) and substance use.

\section{Materials and Methods}

\subsection{Population, Sampling Design and Representativeness}

The ESPAD target population is defined as regular students who turn 16 during the calendar year of the survey and are present in the classroom on the day of the survey [36,37]. This definition 
includes students who are enrolled in regular, vocational, general or academic studies but excludes those enrolled in either special schools or special classes for students with learning disorders or severe physical handicaps. Part-time and evening students and military high schools were also excluded. Sampling in the ESPAD project is based on the class as the final sampling unit. A total of 104,828 students participated in the 2007 ESPAD study and 103,076 students in the 2011 study. More details about methodology are available in the ESPAD Reports $[36,37]$.

Among all Romanian inhabitants born in 1991 and 1995, roughly 87\% and 94\%, respectively, were still enrolled in regular schools. The remaining students were enrolled in either a vocational, theological or military school, or in schools where the teaching language is not Romanian.

The Romanian sampling frame included 9th and 10th graders and covered approximately $99 \%$ of the ESPAD target population (the remaining students were in the 8th grade). The sampling frame was nationally representative for students from regular schools and covered all 42 counties.

A simple random sampling procedure was applied to a list of 1459 schools in 2007 and 1499 schools in 2011, in order to obtain an adequate geographical distribution. Both lists were provided by the Ministry of Education. These lists did not include information about school size, meaning that all schools had the same probability of being sampled. From these schools, one class per grade was randomly selected to participate without class size being considered. The samples are representative for Romanian students born in 1991 and 1995 enrolled in grades 9 and 10 at regular schools. Using the detailed information about school and class size provided by the schools contacted, a weight has been introduced to adjust for school size.

\subsection{Organization of the Study}

Once classes had been selected, the parents received information about the study in order to give their active consent; the schools received a folder with methodological information and the headmasters were asked to make plans for the data-collection procedure. The questionnaires and response envelopes were distributed by ordinary post to the research assistants.

Research assistants collected data in the classrooms where the students answered the questionnaires anonymously. They received standard instructions and individual sealable response envelopes to put their questionnaires in. The completed questionnaires were brought by the research assistants to the county center where the data were entered.

This study was approved by the Research Ethics Committee of Victor Babes University of Medicine and Pharmacy (No. 03/2013).

\subsection{School and Student Participation}

Students and schools were informed that participation in the survey was voluntary. The overall response rate was $84 \%$ in 2007 and $79 \%$ in 2011 . Only $2 \%$ of the research assistants experienced that some of the students found the questionnaire difficult to complete. A total of 2289 Romanian students were included in the final database in 2007 and 2770 in the 2011 database.

\subsection{Instrument, Measurement and Data Processing}

The translation of the questionnaire was made by a team of professional translators, after which it was back-translated and reviewed by a psychiatrist and public-health specialists. The questionnaire was pre-tested at ten schools, which led to some modifications.

Cronbach's alpha reliability coefficient was determined for the main parts of the questionnaire investigating social support, substance use, violence, etc. Results ranging from 0.77 to 0.81 were found, indicating that participants responded consistently to questionnaire items.

Aggressive behaviors were assessed through the following questions: How many times during the last 12 months he/she had: experienced a physical fight, hit one of the teachers, got mixed into a fight at school or at work, took part in a fight where a group of friends were against another group, hurt somebody badly enough to need bandages or a doctor, used any kind of weapon to 
get something from a person, participated in a group teasing an individual, participated in a group bruising an individual, participated in a group starting a fight with another group, started a fight with another individual.

Victimization was assessed through questions like: How many times during the past 12 months have you been: individually teased by a whole group of people, bruised by a whole group of people, in a group that was attacked by another group, or individually involved in a fight started by someone else.

The use of tobacco, alcohol and illicit drugs was assessed through questions that aimed to establish whether these substances have ever been used by the participants, age of first use and the possibility of consumption during the past 30 days. Binge drinking was assessed by asking how many days out of the last 30 the respondent had five or more drinks in a row.

The answers for all the question from above were dichotomized to not at all and once or more times.

Relationship with parents and perceived parental behavior were assessed as follows: relationships with parents (satisfied, neither nor, not satisfied, not at all satisfied, there is no such person); family control was assessed by the questions "Do your parent(s) set definite rules about what you can do at home?", "Do your parent(s) set definite rules about what you can do outside the home?", "Do your parent(s) know whom you are spending your evenings with?", " Do your parent(s) know where you are in the evenings?" (almost always, often, sometimes, seldom, almost never), "Do your parent(s) know where you spend Saturday nights?" (always, quite often, sometimes, usually do not know); emotional support and caring from mother and/or father (almost always, often, sometimes, seldom, almost never).

One item analyzed relationship with friends (satisfied, neither nor, not satisfied, not so satisfied, not at all satisfied, there is no such person) and two items assessed emotional support and caring from best friend (almost always, often, sometimes, seldom, almost never).

Students were also asked about their school performance, mainly their grades at the end of the last term and about absenteeism during last 30 days.

The variable, "How often during the last 12 months have you experienced physical fight?" was dichotomized and the new variable was grouped as follows: never/one or more physical fights during the past 12 months. This question was introduced for the first time in the 2011 survey.

The data were entered manually in each county during a five-week period and then centrally merged by the National School of Public Health, Management and Professional Development, Bucharest, Romania.

\subsection{Statistical Analysis}

All analyses were performed on weighted data. The results are presented as absolute and relative frequencies. All analyses were conducted with Stata 9.2 (Statacorp, Texas, TX, USA) using the svy commands. Descriptive statistics were conducted using frequencies and proportions. Chi-square tests were performed to compare values between 2007 and 2011. A logistic regression analysis was used to estimate factors associated with physical fight experienced during previous 12 months. A $p<0.05$ was considered statistically significant, and odds ratios (OR) with their respective $95 \%$ confidence interval (CI) were calculated.

\section{Results}

A total of 2289 students ( 1009 males $-44.08 \%$ and 1280 females- $55.92 \%$ ) were included in the survey in 2007, and 2770 students (1279 males-46.17\% and 1491 females-53.83\%) in 2011. The present study revealed that 1000 students $(35.87 \%$ ) had experienced a physical fight during the previous 12 months. Univariate analysis showed important differences between students who experienced physical fight and those who did not (Table 1). 
Table 1. Factors associated with physical fight experienced by Romanian students during the last 12 months determined by univariate analysis for the 2011 survey.

\begin{tabular}{|c|c|c|c|c|c|}
\hline \multirow[b]{2}{*}{ Types of Factors } & \multirow[b]{2}{*}{ Variables } & \multirow[b]{2}{*}{ OR } & \multicolumn{2}{|c|}{$95 \%$ CI } & \multirow[b]{2}{*}{$p$ Value } \\
\hline & & & $\begin{array}{l}\text { Lower } \\
\text { Limit }\end{array}$ & $\begin{array}{l}\text { Upper } \\
\text { Limit }\end{array}$ & \\
\hline \multirow{3}{*}{ Demographic } & Gender & & & & \\
\hline & Female & 1 Ref & & & \\
\hline & Male & 1.95 & 1.67 & 2.29 & $<0.001$ \\
\hline \multirow[t]{43}{*}{ Social: parents } & Relationship with mother & & & & $<0.001$ \\
\hline & Very satisfied & 1 Ref & & & \\
\hline & Satisfied & 1.50 & 1.25 & 1.79 & $<0.001$ \\
\hline & Neither nor & 1.94 & 1.37 & 2.76 & $<0.001$ \\
\hline & Not so satisfied & 1.31 & 0.91 & 1.87 & 0.142 \\
\hline & Not at all satisfied & 1.95 & 1.09 & 3.47 & 0.021 \\
\hline & There is no such person & 6.36 & 2.29 & 17.68 & $<0.001$ \\
\hline & Relationship with father & & & & $<0.001$ \\
\hline & Very satisfied & 1 Ref & & & \\
\hline & Satisfied & 1.33 & 1.10 & 1.60 & 0.002 \\
\hline & Neither nor & 1.57 & 1.14 & 2.16 & 0.005 \\
\hline & Not so satisfied & 1.63 & 1.21 & 2.20 & 0.001 \\
\hline & Not at all satisfied & 1.72 & 1.16 & 2.54 & 0.006 \\
\hline & There is no such person & 1.69 & 1.07 & 2.68 & 0.022 \\
\hline & $\begin{array}{l}\text { Parents know where } \\
\text { adolescents spend their } \\
\text { Saturday nights }\end{array}$ & & & & $<0.001$ \\
\hline & Always & 1 Ref & & & \\
\hline & Quite often & 1.99 & 1.64 & 2.42 & $<0.001$ \\
\hline & Sometimes & 2.84 & 2.22 & 3.36 & $<0.001$ \\
\hline & Usually do not know & 2.74 & 1.86 & 4.03 & $<0.001$ \\
\hline & $\begin{array}{l}\text { Parents know with whom } \\
\text { adolescents are with in the } \\
\text { evenings }\end{array}$ & & & & $<0.001$ \\
\hline & Almost always & 1 Ref & & & \\
\hline & Often & 2.10 & 1.68 & 2.62 & $<0.001$ \\
\hline & Sometimes & 2.52 & 1.95 & 3.26 & $<0.001$ \\
\hline & Seldom & 3.30 & 2.46 & 4.41 & $<0.001$ \\
\hline & Almost never & 2.01 & 1.54 & 2.63 & $<0.001$ \\
\hline & $\begin{array}{l}\text { Parents know where } \\
\text { adolescents are in the } \\
\text { evenings }\end{array}$ & & & & $<0.001$ \\
\hline & Almost always & 1 Ref & & & \\
\hline & Often & 1.98 & 1.59 & 2.47 & $<0.001$ \\
\hline & Sometimes & 2.47 & 1.90 & 3.23 & $<0.001$ \\
\hline & Seldom & 3.60 & 2.64 & 4.90 & $<0.001$ \\
\hline & Almost never & 1.92 & 1.43 & 2.58 & $<0.001$ \\
\hline & Caring from parents & & & & $<0.001$ \\
\hline & Almost always & 1 Ref & & & \\
\hline & Often & 1.77 & 1.45 & 2.15 & $<0.001$ \\
\hline & Sometimes & 1.86 & 1.46 & 2.35 & $<0.001$ \\
\hline & Seldom & 2.66 & 1.90 & 3.74 & $<0.001$ \\
\hline & Almost never & 2.14 & 1.47 & 3.11 & $<0.001$ \\
\hline & $\begin{array}{l}\text { Emotional support from } \\
\text { parents }\end{array}$ & & & & $<0.001$ \\
\hline & Almost always & 1 Ref & & & \\
\hline & Often & 1.72 & 1.41 & 2.10 & $<0.001$ \\
\hline & Sometimes & 1.87 & 1.48 & 2.38 & $<0.001$ \\
\hline & Seldom & 2.01 & 1.43 & 2.82 & $<0.001$ \\
\hline & Almost never & 1.86 & 1.27 & 2.72 & 0.001 \\
\hline
\end{tabular}


Table 1. Cont.

\begin{tabular}{|c|c|c|c|c|c|}
\hline \multirow[b]{2}{*}{ Types of Factors } & \multirow[b]{2}{*}{ Variables } & \multirow[b]{2}{*}{ OR } & \multicolumn{2}{|c|}{$95 \%$ CI } & \multirow[b]{2}{*}{$p$ Value } \\
\hline & & & $\begin{array}{l}\text { Lower } \\
\text { Limit }\end{array}$ & $\begin{array}{l}\text { Upper } \\
\text { Limit }\end{array}$ & \\
\hline & $\begin{array}{l}\text { Serious problems with } \\
\text { parents }\end{array}$ & & & & $<0.001$ \\
\hline & No & 1 Ref & & & \\
\hline & Yes & 2.02 & 1.71 & 2.39 & $<0.001$ \\
\hline \multirow{22}{*}{ Social: friends } & Relationship with friends & & & & 0.001 \\
\hline & Very satisfied & 1 Ref & & & \\
\hline & Satisfied & 1.15 & 0.98 & 1.36 & 0.086 \\
\hline & Neither nor & 1.70 & 1.20 & 2.40 & 0.002 \\
\hline & Not so satisfied & 1.14 & 0.75 & 1.73 & 0.537 \\
\hline & Not at all satisfied & 2.30 & 1.02 & 5.20 & 0.038 \\
\hline & There is no such person & 3.90 & 0.71 & 21.44 & 0.091 \\
\hline & Caring from best friend & & & & $<0.001$ \\
\hline & Almost always & 1 Ref & & & \\
\hline & Often & 1.26 & 1.03 & 1.54 & 0.022 \\
\hline & Sometimes & 1.29 & 1.03 & 1.60 & 0.022 \\
\hline & Seldom & 1.62 & 1.22 & 2.16 & $<0.001$ \\
\hline & Almost never & 1.38 & 0.97 & 1.95 & 0.068 \\
\hline & $\begin{array}{l}\text { Emotional support from } \\
\text { best friend }\end{array}$ & & & & 0.001 \\
\hline & Almost always & 1 Ref & & & \\
\hline & Often & 1.46 & 1.19 & 1.78 & $<0.001$ \\
\hline & Sometimes & 1.24 & 0.99 & 1.54 & 0.051 \\
\hline & Seldom & 1.62 & 1.18 & 2.22 & 0.002 \\
\hline & Almost never & 1.39 & 0.98 & 1.97 & 0.062 \\
\hline & $\begin{array}{l}\text { Serious problems with } \\
\text { friends }\end{array}$ & & & & $<0.001$ \\
\hline & No & 1 Ref & & & \\
\hline & Yes & 2.11 & 1.79 & 2.48 & $<0.001$ \\
\hline \multirow[t]{14}{*}{ School performance } & Grades & & & & $<0.001$ \\
\hline & 9-10 (highest) & 1 Ref & & & \\
\hline & $8-8.99$ & 1.82 & 1.46 & 2.27 & $<0.001$ \\
\hline & $7-7.99$ & 2.50 & 1.98 & 3.15 & $<0.001$ \\
\hline & $6-6.99$ & 3.35 & 2.50 & 4.49 & $<0.001$ \\
\hline & $5-5.99$ & 3.02 & 2.02 & 4.52 & $<0.001$ \\
\hline & $<5$ & 1.09 & 0.29 & 4.09 & 0.892 \\
\hline & $\begin{array}{l}\text { Skipped classes last } 30 \\
\text { days }\end{array}$ & & & & $<0.001$ \\
\hline & None & 1 Ref & & & \\
\hline & 1 day & 1.81 & 1.48 & 2.21 & $<0.001$ \\
\hline & 2 days & 2.57 & 1.96 & 3.36 & $<0.001$ \\
\hline & 3-4 days & 3.39 & 2.46 & 4.67 & $<0.001$ \\
\hline & 5-6 days & 5.97 & 3.44 & 10.37 & $<0.001$ \\
\hline & $\geq 7$ days & 6.24 & 3.98 & 9.80 & $<0.001$ \\
\hline \multirow[t]{9}{*}{ Substance use } & Marijuana lifetime use & & & & $<0.001$ \\
\hline & No & 1 Ref & & & \\
\hline & Yes & 2.78 & 2.06 & 3.75 & $<0.001$ \\
\hline & Current smoking & & & & $<0.001$ \\
\hline & No & 1 Ref & & & \\
\hline & Yes & 2.46 & 2.07 & 2.92 & $<0.001$ \\
\hline & Binge drinking last 30 days & & & & $<0.001$ \\
\hline & No & 1 Ref & & & \\
\hline & Yes & 2.91 & 2.46 & 3.45 & $<0.001$ \\
\hline
\end{tabular}


The following variables were significant factors associated with physical fighting: male gender, poor relationships with mother and father, parent(s) do not know where adolescents spend their Saturday nights, parent(s) do not know where and with whom the adolescents spend their evenings, poor caring and emotional support from parent(s), serious problems with parents, poor caring and emotional support from best friend, serious problems with friends, low school grades, high truancy, marijuana lifetime use, current smoking and binge drinking during the previous 30 days.

We did not find any association between physical fight experienced during the last 12 months and definite rules set by parent(s) regarding what adolescents can do at home and outside home. Using stepwise logistic regression, the most parsimonious multivariate logistic model was produced for factors associated with physical fight experienced during the last 12 months (Table 2).

Table 2. Factors associated with physical fight experienced by Romanian students during the last 12 months determined by multivariate analysis for the 2011 survey.

\begin{tabular}{|c|c|c|c|c|c|}
\hline \multirow[b]{2}{*}{ Types of Factors } & \multirow[b]{2}{*}{ Variables } & \multirow[b]{2}{*}{ OR * } & \multicolumn{2}{|c|}{$95 \% \mathrm{CI}$} & \multirow[b]{2}{*}{$p$ Value } \\
\hline & & & $\begin{array}{l}\text { Lower } \\
\text { Limit }\end{array}$ & $\begin{array}{l}\text { Upper } \\
\text { Limit }\end{array}$ & \\
\hline \multirow[t]{3}{*}{ Demographic } & Gender & & & & $<0.001$ \\
\hline & Female & 1 Ref & & & \\
\hline & Male & 1.79 & 1.39 & 2.29 & \\
\hline \multirow[t]{12}{*}{ Social: parents } & $\begin{array}{l}\text { Parents know with whom } \\
\text { adolescents are in the } \\
\text { evenings }\end{array}$ & & & & $<0.001$ \\
\hline & Almost always & 1 Ref & & & \\
\hline & Often & 1.64 & 1.19 & 2.26 & 0.002 \\
\hline & Sometimes & 1.76 & 1.12 & 2.73 & 0.011 \\
\hline & Seldom & 2.22 & 1.47 & 3.36 & $<0.001$ \\
\hline & Almost never & 1.42 & 0.88 & 2.31 & 0.146 \\
\hline & Caring from parents & & & & $<0.001$ \\
\hline & Almost always & 1 Ref & & & \\
\hline & Often & 1.26 & 0.95 & 1.68 & 0.113 \\
\hline & Sometimes & 1.40 & 0.96 & 2.05 & 0.082 \\
\hline & Seldom & 2.63 & 1.57 & 4.41 & $<0.001$ \\
\hline & Almost never & 2.17 & 0.88 & 5.39 & 0.085 \\
\hline \multirow[t]{3}{*}{ Social: friends } & $\begin{array}{l}\text { Serious problems with } \\
\text { friends }\end{array}$ & & & & $<0.001$ \\
\hline & No & 1 Ref & & & \\
\hline & Yes & 1.75 & 1.37 & 2.24 & $<0.001$ \\
\hline \multirow[t]{14}{*}{ School performance } & Grades & & & & $<0.001$ \\
\hline & 9-10 (highest) & 1 Ref & & & \\
\hline & $8-8.99$ & 1.44 & 1.07 & 1.94 & 0.016 \\
\hline & $7-7.99$ & 1.68 & 1.19 & 2.38 & 0.002 \\
\hline & $6-6.99$ & 2.16 & 1.29 & 3.62 & 0.002 \\
\hline & $5-5.99$ & 0.67 & 0.30 & 1.48 & 0.320 \\
\hline & $<5$ & 3.75 & 0.08 & 179.89 & 0.472 \\
\hline & $\begin{array}{l}\text { Skipped classes last } 30 \\
\text { days }\end{array}$ & & & & $<0.001$ \\
\hline & None & 1 Ref & & & \\
\hline & 1 day & 1.46 & 1.13 & 1.89 & 0.004 \\
\hline & 2 days & 1.70 & 1.14 & 2.52 & 0.008 \\
\hline & 3-4 days & 1.97 & 1.23 & 3.15 & 0.004 \\
\hline & 5-6 days & 6.71 & 2.20 & 20.44 & $<0.001$ \\
\hline & $\geq 7$ days & 3.28 & 1.72 & 6.27 & $<0.001$ \\
\hline \multirow[t]{3}{*}{ Substance use } & Binge drinking last 30 days & & & & $<0.001$ \\
\hline & No & 1 Ref & & & \\
\hline & Yes & 2.08 & 1.60 & 2.70 & $<0.001$ \\
\hline
\end{tabular}


The following factors associated with physical fight remained: male gender, parent(s) do not know where and with whom the adolescents spend their evenings, poor parental caring, serious problems with friends, low school grades, high truancy and binge drinking during the previous 30 days. A decrease in almost all aggressive behaviors was noticed in 2011 compared to 2007 (Table 3).

Table 3. Violent behavior: 2007 compared to 2011 among Romanian students.

\begin{tabular}{|c|c|c|c|c|c|c|}
\hline \multirow{2}{*}{$\begin{array}{c}\text { Year } \\
\text { Characteristic }\end{array}$} & \multicolumn{2}{|c|}{2007} & \multicolumn{2}{|c|}{2011} & \multicolumn{2}{|c|}{ Significance } \\
\hline & $\begin{array}{c}\text { Not at all, } n \\
(\%)\end{array}$ & $\begin{array}{l}\text { Once or more } \\
\text { Times, } n(\%)\end{array}$ & $\begin{array}{c}\text { Not at all, } n \\
(\%)\end{array}$ & $\begin{array}{l}\text { Once or more } \\
\text { Times, } n(\%)\end{array}$ & $\begin{array}{c}\text { Chi } \\
\text { Square }\end{array}$ & $p$ Value \\
\hline Hit teacher & $2232(98.20)$ & $42(1.80)$ & $2685(98.38)$ & $52(1.62)$ & 0.018 & 0.891 \\
\hline Males & $963(96.70)$ & $36(3.30)$ & $1223(97.53)$ & $35(2.47)$ & 1.233 & 0.267 \\
\hline Females & $1269(99.54)$ & $6(0.46)$ & $1462(99.06)$ & $17(0.94)$ & 3.810 & 0.051 \\
\hline $\begin{array}{l}\text { Got mixed into a fight where } \\
\text { a group of friends were } \\
\text { against another group }\end{array}$ & $1853(81.63)$ & 408 (18.37) & $2273(83.49)$ & $454(16.51)$ & 1.687 & 0.194 \\
\hline Males & $740(74.25)$ & $251(25.75)$ & 955 (76.71) & $297(23.29)$ & 0.772 & 0.379 \\
\hline Females & $1113(88.28)$ & 157 (11.72) & $1318(89.0)$ & $157(11.0)$ & 1.988 & 0.158 \\
\hline $\begin{array}{l}\text { Participated into a group } \\
\text { fight }\end{array}$ & $1763(77.16)$ & $503(22.84)$ & $2239(82.86)$ & $470(17.14)$ & 18.434 & $<0.001$ \\
\hline Males & 707 (70.03) & $285(29.97)$ & $943(76.0)$ & $298(24.0)$ & 6.358 & 0.012 \\
\hline Females & $1056(83.53)$ & $218(16.47)$ & $1296(88.39)$ & 172 (11.61) & 16.271 & $<0.001$ \\
\hline Hurt someone badly & $2070(90.57)$ & $196(9.43)$ & $2504(92.23)$ & $221(7.77)$ & 0.470 & 0.493 \\
\hline Males & $862(85.73)$ & $132(14.27)$ & 1093 (87.69) & $158(12.31)$ & 0.207 & 0.648 \\
\hline Females & $1208(94.93)$ & $64(5.07)$ & $1411(95.91)$ & $63(4.09)$ & 0.887 & 0.346 \\
\hline Weapon use & $2223(98.13)$ & $42(1.87)$ & $2659(97.79)$ & $70(2.21)$ & 2.851 & 0.091 \\
\hline Males & $960(96.48)$ & $35(3.52)$ & $1204(96.46)$ & $50(3.54)$ & 0.336 & 0.562 \\
\hline Females & 1263 (99.62) & $7(0.38)$ & 1455 (98.87) & $20(1.13)$ & 4.537 & 0.033 \\
\hline Group teasing & $1119(47.16)$ & $1152(52.84)$ & $1511(54.07)$ & $1230(45.93)$ & 17.057 & $<0.001$ \\
\hline Males & $480(44.09)$ & $521(55.91)$ & $689(53.74)$ & $572(46.26)$ & 9.992 & 0.002 \\
\hline Females & 639 (49.93) & $631(50.07)$ & 822 (54.35) & $658(45.65)$ & 7.494 & 0.006 \\
\hline Group bruising & $1728(75.55)$ & $537(24.45)$ & $2134(77.98)$ & $601(22.02)$ & 2.119 & 0.145 \\
\hline Males & 731 (72.09) & $265(27.91)$ & $938(74.4)$ & $321(25.61)$ & 0.356 & 0.551 \\
\hline Females & 997 (78.68) & $272(21.32)$ & $1196(80.90)$ & $280(19.1)$ & 2.578 & 0.108 \\
\hline Gang attacking & $1764(78.03)$ & $499(21.97)$ & $2230(81.72)$ & $503(18.28)$ & 10.262 & 0.001 \\
\hline Males & $742(74.01)$ & $252(25.99)$ & $963(76.55)$ & $293(23.45)$ & 1.238 & 0.266 \\
\hline Females & $1022(81.66)$ & 247 (18.34) & $1267(85.92)$ & $210(14.08)$ & 13.541 & $<0.001$ \\
\hline Individual attacking & $1620(70.80)$ & $629(29.20)$ & $2178(80.31)$ & $546(19.69)$ & 42.865 & $<0.001$ \\
\hline Males & $614(60.22)$ & $374(39.78)$ & $917(73.45)$ & $335(26.55)$ & 31.434 & $<0.001$ \\
\hline Females & $1006(80.32)$ & 255 (19.68) & $1261(85.88)$ & $211(14.12)$ & 16.646 & $<0.001$ \\
\hline Group teased & $1248(53.15)$ & $1016(46.85)$ & $1732(63.04)$ & $1004(36.96)$ & 34.430 & $<0.001$ \\
\hline Males & $515(48.84)$ & $481(51.16)$ & $783(62.80)$ & $478(37.20)$ & 24.568 & $<0.001$ \\
\hline Females & 733 (57.03) & 535 (42.97) & 949 (63.23) & $526(36.77)$ & 12.263 & $<0.001$ \\
\hline Group bruised & $1953(85.56)$ & $302(14.44)$ & $2431(89.50)$ & $296(10.50)$ & 7.527 & 0.006 \\
\hline Males & 787 (78.02) & $204(21.98)$ & $1065(85.69)$ & $192(14.31)$ & 10.769 & 0.001 \\
\hline Females & $1166(92.38)$ & $98(7.62)$ & 1366 (92.62) & $104(7.38)$ & 0.457 & 0.499 \\
\hline Gang attacked & $1856(82.63)$ & $388(17.37)$ & $2358(87.08)$ & $365(12.92)$ & 14.445 & $<0.001$ \\
\hline Males & $757(75.85)$ & $230(24.15)$ & 1035 (83.24) & $215(16.76)$ & 12.890 & $<0.001$ \\
\hline Females & $1099(88.76)$ & $158(11.24)$ & $1323(90.20)$ & $150(9.80)$ & 3.858 & 0.049 \\
\hline Individual attacked & $1571(68.07)$ & $683(31.93)$ & $2122(78.91)$ & $588(21.09)$ & 47.825 & $<0.001$ \\
\hline Males & $574(56.10)$ & $416(43.90)$ & $876(71.22)$ & $371(28.78)$ & 36.427 & $<0.001$ \\
\hline Females & 997 (78.83) & $267(21.17)$ & $1246(85.18)$ & $217(14.82)$ & 18.383 & $<0.001$ \\
\hline
\end{tabular}

Statistically significant differences were observed for: taking part in a fight where a group of friends went against another group, participating in a group teasing an individual, participating in a group starting a fight with another group (only for females), starting a fight with another individual.

The only violent behavior that increased in 2011, compared to 2007, was using any kind of weapon to get something from a person, but this was statistically significant only for females. 
Regarding victimization, we also found a decrease of prevalence in 2011 compared to 2007. Statistically significant differences were observed for: being individually teased by a whole group of people, being bruised by a whole group of people (only for males), being in a group that was attacked by another group, being individually involved in a fight started by someone else.

\section{Discussion}

The ESPAD surveys among high school students provided us an opportunity to study aggressive behavior in two large national samples in 2007 and 2011. The present study revealed that $35.87 \%$ of the students experienced physical fight during the previous 12 months. This result was higher than the prevalence of physical fight for the entire European ESPAD database [38] that was 31.25\%, the lowest value being recorded in Denmark (19.68\%) and the highest in Malta (47.74\%) [37].

The 2011 questionnaire included a new variable regarding the physical fighting experienced by the students during the previous 12 months. We investigated different types of factors associated with physical fighting: demographic (gender), social (relationship with parents, parental control, emotional support and relationship with friends and their support), school performance (grades, skipped classes) and substance use (marijuana use, smoking and binge drinking).

The present study has demonstrated that the combination of male gender, binge drinking during the previous 30 days, having serious problems with friends, parent(s) who do not know where and with whom the adolescents spend their evenings, poor parental caring, low school grades, and high truancy were predictive of physical fighting in this adolescent population. However, the results do not provide information about a causal relationship.

Similar to other studies [16,39-43], physical fighting was more prevalent in boys than in girls. Boys usually engage in undisguised violence to gain influence, money or power. Girls resort to relational aggression and may become violent when it comes to emotional situations, such as peer and/or romantic relationships, family arguments or outsiders' instigation [44-46].

According to our findings, students with poor school performance (grades between 6 and 6.99) were the most exposed to experience physical fighting compared to the highest school performance (grades between 9 and 10). The multivariate analysis showed they were three times more likely to engage in physical fight (OR $=2.16,95 \% \mathrm{CI}: 1.29-3.62, p=0.002)$. This is similar to other studies' findings $[47,48]$.

Absenteeism has also been found to be associated with youth violence $[14,49,50]$. In our logistic regression model, students who have been missing 5-6 days from school in the last 30 days were almost seven times more likely to engage in physical fighting (OR $=6.71,95 \% \mathrm{CI}$ : $2.20-20.44, p<0.001$ ).

The development of bullying and victimization might be influenced by individual and family factors. Aggressive behavior research has shown that children's socialization experiences within the family have a major role in aggressive behaviors development [36]. The following family influences on the development of aggression have been studied: family demographics (income, family type), parenting techniques (punitive and inconsistent discipline), and relationships between parent and child (positive and negative interactions) [37].

We found that the adolescents who are more likely to experience physical fights ( $\mathrm{OR}=2.22,95 \% \mathrm{CI}$ : 1.47-3.36, $p<0.001$ ) usually have parents who rarely know with whom their children spend their evenings. Poor parental care and serious problems with friends were another two important predictors in our model.

Certainly, a wide variety of factors contribute to today's adolescents' exposure to violent behaviors, including family structure, social environment and peer behavior. Two of the most common correlates of violent behavior are alcohol and drug use [51,52]. For example, alcohol may suppress inhibitions against violent behavior or may affect the brain in such a way as to produce aggressive behaviors $[53,54]$. A competing theory proposes the reverse causal relationship, i.e., people who plan on being violent may drink to give themselves courage or an excuse for the violence [55-57]. Finally, a third theory states that drug use, alcohol use and violence are all outcomes of an unobserved third factor, for example, 
a risk-taking personality $[39,51,58]$. Risk taking is frequent during adolescence, and is associated with adverse outcomes including substance use. It is likely to be influenced by an individual's cognitive development, social development, and experiences with dangerous situations [59]. The inability to recognize warning signs in dangerous situations can make drinkers easy targets for perpetrators [52]. Our study showed that alcohol consumption and drug use is a significant predictor for developing an aggressive behavior.

We observed declines in almost all aggressive behaviors in 2011 compared to 2007. This trend is also confirmed by the two HBSC studies that took place in the same periods in Romania [32,33]. After gaining access to the EU in January 2007, new legislation has been enacted and previous rules have been reinforced in areas related to youth violence. For instance, the Romanian Parliament adopted two laws on improving safety in schools: Law No. 35/2007 and Law No. 29/2010 amending and supplementing Law No. 35/2007 [60,61]. This new legislation may have played a role in reducing violence, but there is no proof of causality.

\section{Strengths and Limitations of the Study}

There are certain limitations of this study that must be considered when interpreting the results. First, the findings reported here are only relevant to high school students from Romania who turn 16 in the calendar year of the survey and may not be generalized to other adolescents in the same age group that are not included in scholastic institutions. Second, survey methods are frequently criticized because they rely on the validity of self-reporting of sensitive and highly stigmatized behavior, thus error based on self-reported behavior might have been generated. Third, adolescents who were not available to complete the questionnaire due to truancy or dropout are likely to be at higher risk for aggressive behavior and other risk behaviors. Despite the limitations mentioned above, the study has strengths. We used a standardized questionnaire employed in other European countries in similar settings. The prevalence estimates we obtained are likely to closely represent the aggressive behavior prevalence amongst adolescents going to school, as we used probability methods for selecting the sample.

\section{Conclusions}

Physical fighting amongst the young adolescents that we evaluated was higher than the prevalence of physical fighting for the entire European ESPAD database [38], and was associated with several factors. A combination between male gender, binge drinking, problematic relationships with friends and family members, low school grades, absenteeism was found to be associated to the violent behaviors of adolescents. The development of a theoretical model which separates problem behaviors from adolescent experimental or risk-taking behaviors might be useful for future evaluations. The novelty of this study lies in analyzing patterns of associations, using a large sample with national representation. These findings may be useful to support and guide policy makers regarding the improvement and implementation of strategies to further prevent aggressive behaviors in teenagers. As in other European countries, Romania managed to reduce aggressive behaviors among high school students. New legislation may have played a role in reducing violence, but there is no proof of causality. The Ministry of Education encouraged the development of partnerships between representatives of the County School Inspectorates and the County Police Inspectorates to fight against violence in schools. In addition, the increase of alcohol excise played an important role, especially for children with limited access to their parents' funds; this was coupled with the banning of alcohol advertising and clear rules for TV content for children and youth. Concomitantly, various guides concerning violence prevention in school were published. A school intervention strategy must provide a detailed presentation of the objectives pursued, including the expected results, the activities to be carried out, the actors involved and their responsibilities, the time horizon, the necessary resources, monitoring and evaluation modalities. These interventions should provide students and teachers information about violence, change the way adolescents feel and think about it, and teach non-violent skills in order to resolve disputes. Skill enhancement training with parents could be an important factor 
in controlling violence and creating a stronger family bond. Parent-skill and family-relationship approaches, providing caregivers with support and teaching communication skills, might offer problem-solving techniques and behavior-management skills. Additionally, the school psychologists should provide therapy sessions for students in order to strengthen their problem-solving skills and resistance to negative peer influence.

Author Contributions: Conceptualization, S.F. and C.V.; Formal analysis, A.B., S.U., C.C.-B., S.F. and C.V.; Investigation, A.B., C.C.-B., V.C., S.F. and C.V.; Methodology, A.B., S.U., V.C., S.F. and C.V.; Writing-original draft, A.B.; Writing-review \& editing, S.U., C.C.-B., V.C., S.F. and C.V. All authors have read and agreed to the published version of the manuscript.

Funding: This research received no external funding.

Acknowledgments: The authors would like to acknowledge the members of the ESPAD group who collected the national data (http://www.espad.org/report/acknowledgements) and the funding bodies who supported the international coordination of ESPAD: the government of Sweden, the EMCDDA and the Pompidou Group. Special thanks are due to the schoolchildren, teachers and national funding bodies who made this project possible.

Conflicts of Interest: The authors declare no conflict of interest.

\section{References}

1. Boulton, M.J.; Underwood, K. Bully/victim problems among middle school children. Br. J. Educ. Psychol. 1992, 62, 73-87. [CrossRef] [PubMed]

2. Craig, W.; Harel-Fisch, Y.; Fogel-Grinvald, H.; Dostaler, S.; Hetland, J.; Simons-Morton, B.; Molcho, M.; de Mato, M.G.; Overpeck, M.; Due, P.; et al. A cross-national profile of bullying and victimization among adolescents in 40 countries. Int. J. Public Health 2009, 54, 216-224. [CrossRef] [PubMed]

3. Craig, W.M.; Pepler, D.J. Identifying and targeting risk for involvement in bullying and victimization. Can. J. Psychiatry 2003, 48, 577-582. [CrossRef] [PubMed]

4. Due, P.; Holstein, B.E.; Lynch, J.; Diderichsen, F.; Gabhain, S.N.; Scheidt, P.; Currie, C. Bullying and symptoms among school-aged children: International comparative cross sectional study in 28 countries. Eur. J. Public Health 2005, 15, 128-132. [CrossRef]

5. Nansel, T.R.; Overpeck, M.D.; Haynie, D.L.; Ruan, W.J.; Scheidt, P.C. Relationships between bullying and violence among US youth. Arch. Pediatr. Adolesc. Med. 2003, 157, 348-453. [CrossRef]

6. Olweus, D. Bully/victim problems among school children: Some basic facts and effects of a schoolbased intervention program. In The Development and Treatment of Childhood Aggression; Pepler, D., Rubin, K., Eds.; Erlbaum: Hillsdale, NJ, USA, 1991; pp. 411-448.

7. Rigby, K.; Slee, P.T. Bullying among Australian School Children: Reported Behavior and Attitudes toward Victims. J. Soc. Psychol. 1991, 131, 615-627. [CrossRef]

8. Smith, P.K.; Sharpe, S. The problem of school bullying. In School Bullying; Smith, P.K., Sharpe, S., Eds.; Routledge: London, UK, 1994; pp. 1-19.

9. Alikasifoglu, M.; Erginoz, E.; Ercan, O.; Uysal, O.; Kaymak, D.A.; Liter, O. Violent behaviour among Turkish high school students and correlates of physical fighting. Eur. J. Public Health 2004, 14, 173-177. [CrossRef]

10. Olweus, D. School-yard bullying-Grounds for intervention. Sch. Saf. 1987, 6, 4-11.

11. Rappaport, N.; Thomas, C. Recent research findings on aggressive and violent behavior in youth: Implications for clinical assessment and intervention. J. Adolesc. Health 2004, 35, 260-277. [CrossRef]

12. Sousa, S.; Correia, T.; Ramos, E.; Fraga, S.; Barros, H. Violence in adolescents: Social and behavioural factors. Gac. Sanit. 2010, 24, 47-52. [CrossRef]

13. Inchley, J.; Currie, D.; Young, T.; Samdal, O.; Torsheim, T.; Augustson, L.; Mathison, F.; Aleman-Diaz, A.; Molcho, M.; Weber, M.; et al. Growing up Unequal: Gender and Socioeconomic Differences in Young People's Health and Well-Being. Health Behavior in School-Aged Children (HBSC) Study: International Report from the 2013/2014 Survey. Health Policy for Children and Adolescents; WHO Regional Office for Europe: Copenhagen, Denmark, 2016.

14. Celedonia, K.L.; Wilson, M.L.; El Gammal, H.A.; Hagras, A.M. Physical fighting among Egyptian adolescents: Social and demographic correlates among a nationally representative sample. Peer J. 2013, 1, e125. [CrossRef] [PubMed] 
15. Fraga, S.; Ramos, E.; Dias, S.; Barros, H. Physical fighting among school-going Portuguese adolescents: Social and behavioural correlates. Prev. Med. 2011, 52, 401-404. [CrossRef]

16. Rudatsikira, E.S.; Kazembe, L.N.; Muula, A.S. Prevalence and associated factors of physical fighting among school-going adolescents in Namibia. Ann. Gen. Psychiatry 2007, 6, 18. [CrossRef] [PubMed]

17. Herrenkohl, T.I.; Huang, B.; Kosterman, R.; Hawkins, J.D.; Catalano, R.F.; Smith, B.H. A comparison of social developmental processes leading to violent behavior in late adolescence for childhood initiators and adolescent initiators of violence. J. Res. Crime Delinq. 2001, 38, 45-63. [CrossRef]

18. Hawkins, J.D.; Weiss, J.G. The Social Development Model: An integrated approach to delinquency prevention. J. Prim. Prev. 1985, 6, 73-97. [CrossRef] [PubMed]

19. Riner, M.E.; Saywell, R.M. Development of the Social Ecology Model of Adolescent Interpersonal Violence Prevention (SEMAIVP). J. Sch. Health 2002, 72, 65-70. [CrossRef] [PubMed]

20. Shetgiri, R.; Kataoka, S.; Ponce, N.; Flores, G.; Chung, P.J. Adolescent fighting: Racial/ethnic disparities and the importance of families and schools. Acad. Pediatr. 2010, 10, 323-329. [CrossRef]

21. Resnick, M.D.; Harris, L.J.; Blum, R.W. The impact of caring and connectedness on adolescent health and well-being. J. Paediatr. Child Health 1993, 29, s1-s9. [CrossRef]

22. Cheng, T.L.; Schwarz, D.; Brenner, R.A.; Wright, J.L.; Fields, C.B.; O’Donnell, R.; Rhee, P.; Scheidt, P.C. Adolescent assault injury: Risk and protective factors and locations of contact for intervention. Pediatrics 2003, 112, 931-938. [CrossRef]

23. Fein, J.A.; Mollen, C.J. Interpersonal violence. Curr. Opin. Pediatr. 1999, 11, 588-593. [CrossRef]

24. Laufer, A.; Harel, Y. The role of family, peers and school perceptions in predicting involvement in youth violence. Int. J. Adolesc. Med. Health 2003, 15, 235-244. [CrossRef] [PubMed]

25. Shetgiri, R.; Lee, S.C.; Tillitski, J.; Wilson, C.; Flores, G. Why adolescents fight: A qualitative study of youth perspectives on fighting and its prevention. Acad. Pediatr. 2015, 15, 103-110. [CrossRef] [PubMed]

26. Di Clemente, R.J.; Hansen, W.B.; Ponton, L.E. (Eds.) Issues in Clinical Child Psychology. Handbook of Adolescent Health Risk Behavior; Plenum Press: New York, NY, USA, 1996.

27. Marlatt, G.A.; VandenBos, G.R. (Eds.) Addictive Behaviors: Readings on Etiology, Prevention, and Treatment; American Psychological Association: Washington, DC, USA, 1997.

28. Wills, T.A. Adolescent Health and Health Behaviors. In International Encyclopedia of the Social E Behavioral Sciences; Smelser, N.J., Baltes, P.B., Eds.; Elsevier: Amsterdam, The Netherlands, 2001.

29. Craig, W.M. The relationship among bullying, victimization, depression, anxiety, and aggression in elementary school children. Personal. Individ. Differ. 1998, 24, 123-130. [CrossRef]

30. Farrington, D.P. Understanding and preventing bullying. In Crime and Justice; Tonry, M., Morris, N., Eds.; University of Chicago Press: Chicago, IL, USA, 1993; pp. 381-458.

31. Espelage, D.; Anderman, E.M.; Brown, V.E.; Jones, A.; Lane, K.L.; McMahon, S.D.; Reddy, L.A.; Reynolds, C.R. Understanding and preventing violence directed against teachers: Recommendations for a national research, practice, and policy agenda. Am. Psychol. 2013, 68, 75-87. [CrossRef] [PubMed]

32. Currie, C.; Gabhainn, S.N.; Godeau, E.; Roberts, C.; Smith, R.; Currie, D.; Pickett, W.; Richter, M.; Morgan, A.; Barnekow, V. Inequalities in Young People's Health: HBSC International Report from the 2005/2006 Survey; WHO Regional Office for Europe: Copenhagen, Denmark, 2008.

33. Currie, C.; Zanotti, C.; Morgan, A.; Currie, D.; de Looze, M.; Roberts, C.; Samdal, O.; Barnekow, V. Social determinants of health and well-being among young people. In Health Behaviour in School-Aged Children (HBSC) Study: International Report from the 2009/2010 Survey; WHO Regional Office for Europe: Copenhagen, Denmark, 2012.

34. Radu, C.P.; Chiriac, D.N.; Vladescu, C. Changing patient classification system for hospital reimbursement in Romania. Croat. Med. J. 2010, 51, 250-258. [CrossRef]

35. Vladescu, C.A.; Scintee, S.G. A Health System Focused on Citizen's Needs. Romania. Hospital Services, Primary Health Care and Human Resources. Rev. Rom. Bioet. 2010, 8, 89-99.

36. Hibell, B.; Guttormsson, U.; Ahlström, S.; Balakireva, O.; Bjarnason, T.; Kokkevi, A.; Kraus, L.; Miller, P.; Plant, M. The 2007 ESPAD Report. Substance Use among Students in 35 European Countries; The Swedish Council for Information on Alcohol and Other Drugs: Stockholm, Sweden, 2009; Available online: https: //www.emcdda.europa.eu/sites/espad.org/files/The_2007_ESPAD_Report-FULL_091006.pdf (accessed on 1 March 2020). 
37. Hibell, B.; Guttormsson, U.; Ahlström, S.; Balakireva, O.; Bjarnason, T.; Kokkevi, A.; Kraus, L. The 2011 ESPAD Report. Substance Use among Students in 36 European Countries; The Swedish Council for Information on Alcohol and Other Drugs: Stockholm, Sweden, 2012; Available online: https://www.emcdda.europa.eu/ sites/espad.org/files/The_2011_ESPAD_Report_FULL_2012_10_29.pdf (accessed on 1 March 2020).

38. ESPAD Database, 2007, 2011. Available online: fttp://www.espad.org/database (accessed on 1 July 2014).

39. Yang, L.; Zhang, Y.; Xi, B.; Bovet, P. Physical fighting and associated factors among adolescents aged 13-15 years in six Western Pacific countries. Int. J. Environ. Res. Public Health 2017, 14, 1427. [CrossRef]

40. Dukes, R.L.; Stein, J.A.; Zane, J.I. Gender differences in the relative impact of physical and relational bullying on adolescent injury and weapon carrying. J. Sch. Psychol. 2010, 48, 511-532. [CrossRef]

41. Pickett, W.; Molcho, M.; Elgar, F.J.; Brooks, F.; de Looze, M.; Rathmann, K.; ter Bogt, T.F.; Nic Gabhainn, S.; Sigmundová, D.; Gaspar de Matos, M.; et al. Trends and socioeconomic correlates of adolescent physical fighting in 30 countries. Pediatrics 2013, 131, e18-e26. [CrossRef]

42. Mat Hussin, S.F.; Abd Aziz, N.S.; Hasim, H.; Sahril, N. Prevalence and factors associated with physical fighting among Malaysian adolescents. Asia Pac. J. Public Health 2014, 26, 108S-115S. [CrossRef]

43. Swahn, M.H.; Gressard, L.; Palmier, J.B.; Yao, H.; Haberlen, M. The prevalence of very frequent physical fighting among boys and girls in 27 countries and cities: Regional and gender differences. J. Environ. Public Health 2013, 2013, 215126. [CrossRef] [PubMed]

44. Finigan-Carr, N.M.; Gielen, A.; Haynie, D.L.; Cheng, T.L. Youth Violence: How Gender Matters in Aggression Among Urban Early Adolescents. J. Interpers. Violence 2016, 31, 3257-3281. [CrossRef] [PubMed]

45. Herrman, J.W.; Silverstein, J. Girls and Violence: A Review of the Literature. J. Community Health Nurs. 2012, 29, 63-74. [CrossRef] [PubMed]

46. Zahn, M.; Brumbaugh, S.; Steffensmeier, D.; Feld, B.; Morash, M.; Chesney-Lind, M.; Miller, J.; Payne, A.A.; Gottfredson, D.C.; Kruttschnitt, C. Violence by Teenage Girls: Trends and Context; US Department of Justice, Office of Juvenile Justice and Delinquency Prevention: Washington, DC, USA, 2008.

47. Borowsky, I.W.; Ireland, M. Predictors of future fight-related injury among adolescents. Pediatrics 2004, 113, 530-536. [CrossRef]

48. Smith-Khuri, E.; Iachan, R.; Scheidt, P.C.; Overpeck, M.D.; Gabhainn, S.N.; Pickett, W.; Harel, Y. A cross-national study of violence-related behaviors in adolescents. Arch. Pediatr. Adolesc. Med. 2004, 158, 539-544. [CrossRef]

49. Acquah, E.O.; Lloyd, J.K.; Davis, L.; Wilson, M.L. Adolescent physical fighting in Ghana, their demographic and social characteristics. Soc. Sci. 2014, 3, 227-241. [CrossRef]

50. Shaikh, M.A.; Abio, A.; Celedonia, K.L.; Lowery Wilson, M. Physical fighting among school-attending adolescents in Pakistan: Associated factors and contextual influences. Int. J. Environ. Res. Public Health 2019, 16, 5039. [CrossRef]

51. Patterson, G.R. Performance models for antisocial boys. Am. Psychol. 1986, 41, 432-444. [CrossRef]

52. World Health Organization. Youth Violence and Alcohol Fact Sheet. Available online: https://www.who.int/ violence_injury_prevention/violence/world_report/factsheets/fs_youth.pdf (accessed on 13 May 2020).

53. Loeber, R.; Stouthamer-Loeber, M. Family factors as correlates and predictors of juvenile conduct problems and delinquency. In Crime and Justice: An Annual Review of Research; Morris, N., Tonry, M., Eds.; University of Chicago Press: Chicago, IL, USA, 1986; Volume 7, pp. 29-149.

54. Markowitz, S. The role of alcohol and drug consumption in determining physical fights and weapon carrying by teenagers. East. Econ. J. 2001, 27, 409-432.

55. Fagan, J. Intoxication and aggression. In Drugs and Crime: Crime and Justice, A Review of Research; Tonry, M., Wilson, J.Q., Eds.; University of Chicago Press: Chicago, IL, USA, 1990; Volume 13, pp. 241-320.

56. Pernanen, K. Theoretical aspects of the relationship between alcohol use and crime. In Drinking and Crime: Perspectives on the Relationships between Alcohol Consumption and Criminal Behaviour; Collins, J., Jr., Ed.; Guilford Press: New York, NY, USA, 1981; pp. 1-69.

57. Reiss, A.J., Jr.; Roth, J.A. Understanding and Preventing Violence: Volume 1; The National Academies Press: Washington, DC, USA, 1993; p. 480.

58. Cordilia, A. Alcohol and property crime: Exploring the causal nexus. J. Stud. Alcohol 1985, 46, $161-171$. [CrossRef] 
59. Dever, B.V.; Schulenberg, J.E.; Dworkin, J.B.; O’Malley, P.M.; Kloska, D.D.; Bachman, J.G. Predicting risk-taking with and without substance use: The effects of parental monitoring, school bonding, and sports participation. Prev. Sci. 2012, 13, 605-615. [CrossRef] [PubMed]

60. LAW no. 35 of March 2, 2007 on increasing safety in educational institutions. In Official Gazette Number 165; 8 March 2007/LEGE nr. 35 din 2 martie 2007 privind creşterea siguranţei în unităţile de învăţământ. În Monitorul Oficial nr. 165; 8 martie 2007. Available online: http://legislatie.just.ro/Public/DetaliiDocument/ 80050 (accessed on 10 April 2020).

61. LAW no. 29 of March 2, 2010 for the amendment and completion of Law no. 35/2007 on increasing safety in educational institutions. In Official Gazette number 143; 4 March 2010/LEGE nr. 29 din 2 martie 2010 pentru modificarea şi completarea Legii nr. 35/2007 privind creşterea siguranţei în unităţile de învăţământ. În Monitorul Oficial nr. 143; 4 martie 2010. Available online: https://lege5.ro/Gratuit/geztenjuhe/legea-nr-29-2010-pentru-modificarea-si-completarea-legii-nr35-2007-privind-cresterea-sigurantei-in-unitatile-de-invatamant (accessed on 10 April 2020).

(C) 2020 by the authors. Licensee MDPI, Basel, Switzerland. This article is an open access article distributed under the terms and conditions of the Creative Commons Attribution (CC BY) license (http://creativecommons.org/licenses/by/4.0/). 